Pourquoi les filles ne sont-elles pas plus nombreuses à choisir une carrière scientifique?

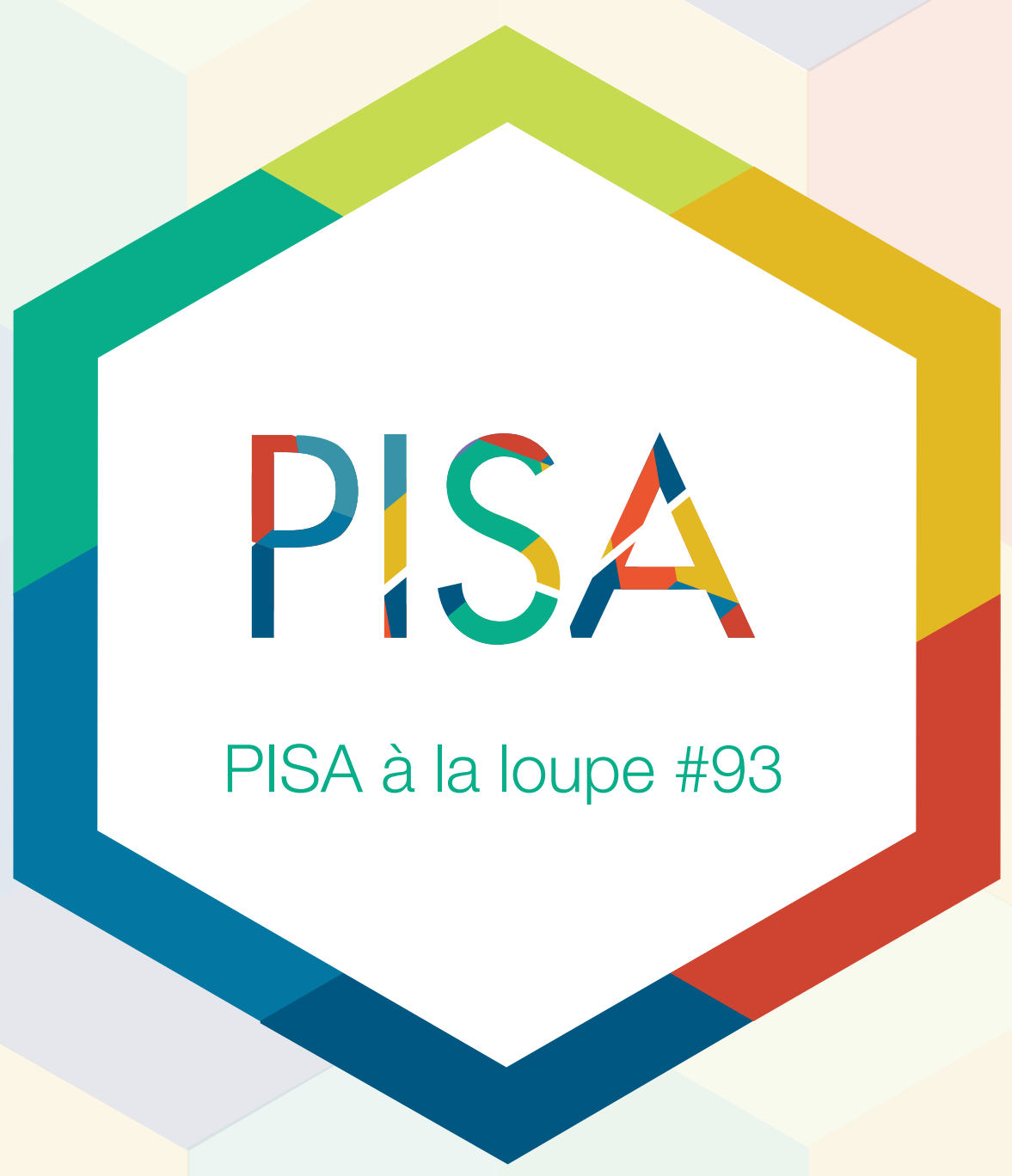




\section{Pourquoi les filles ne sont-elles pas plus nombreuses à choisir une carrière scientifique?}

- Les garçons devancent les filles en sciences dans 22 des pays participant à l'enquête PISA, tandis que c'est l'inverse qui s'observe dans 19 pays. Toutefois, les garçons affichent un point fort relatif en sciences dans 65 des 67 pays participants : ils obtiennent un score en sciences supérieur à leur performance moyenne dans l'ensemble des trois principaux domaines d'évaluation PISA.

- Par rapport aux filles, les garçons font preuve d'une plus grande confiance en leurs capacités d'apprentissage en sciences (dans 39 pays) et d'un intérêt plus marqué pour les sujets scientifiques (dans 51 pays).

- Les différences en faveur des garçons en termes de performance relative, de confiance en leurs capacités et d'intérêt pour les sciences sont associées à de plus faibles taux d'obtention d'un diplôme universitaire en STIM chez les femmes.

Chaque nouvelle publication de données PISA est l'occasion pour des chercheurs du monde entier de se lancer dans de nouvelles analyses afin d'apporter un nouvel éclairage sur des questions de toutes sortes. Parmi celles-ci, l'une reste pour l'instant sans réponse : comment expliquer la sous-représentation des femmes dans les professions en rapport avec les sciences, la technologie, l'ingénierie et les mathématiques (STIM) ? À partir des données du Programme international pour le suivi des acquis des élèves (PISA), Gijsbert Stoet et David Geary se sont intéressés à la nature des écarts entre les sexes dans les domaines des STIM. IIS ont analysé les données de 67 pays et économies ayant participé au cycle 2015 de l'enquête PISA, analyse que sont venus compléter des indicateurs nationaux d'égalité entre les sexes (indice global d'égalité entre les sexes) et le pourcentage de femmes diplômées en STIM. Et leurs travaux se sont avérés des plus fructueux...

\section{L'écart qui s'observe entre les sexes dans les études en STIM est déjà manifeste chez les jeunes de 15 ans.}

Parmi les 67 pays et économies ayant participé à l'enquête PISA 2015, les filles devancent les garçons en sciences dans 19 pays/économies, tandis que c'est l'inverse qui s'observe dans 22 autres. Dans l'ensemble des pays restants, les différences entre les sexes ne sont pas statistiquement significatives. Gijsbert Stoet et David Geary (ci-après «les auteurs ") ont analysé les écarts entre les sexes en examinant la « performance relative » ou le « point fort relatif » de chaque élève dans les trois principaux domaines d'évaluation PISA (voir encadré) ; ils ont alors noté que les filles sont plus fortes en compréhension de l'écrit dans tous les pays, tandis que les garçons sont plus forts en mathématiques dans tous les pays, et en sciences dans 65 des 67 pays/économies à l'étude. En d'autres termes, les garçons obtiennent en sciences et en mathématiques un score supérieur à leur moyenne dans l'ensemble des trois principaux domaines d'évaluation, tandis que les filles obtiennent un score supérieur en compréhension de l'écrit. Ces différences pourraient expliquer pourquoi les garçons sont plus susceptibles que les filles de choisir une carrière dans les domaines des STIM, même si la performance globale des filles et des garçons est similaire : les élèves pourraient en effet choisir leur domaine d'études en fonction de leur point fort relatif, et non absolu. Les filles peuvent être aussi performantes que les garçons en sciences, mais sont en moyenne susceptibles d'être encore meilleures en compréhension de l'écrit.

\section{Les auteurs fondent leur analyse sur trois indicateurs :}

L'indice global d'égalité entre les sexes (données de 2015) évalue la mesure dans laquelle les filles et les femmes accusent un retard par rapport aux garçons et aux hommes pour 14 indicateurs (dont les revenus, le taux de scolarisation dans l'enseignement tertiaire, l'espérance de vie, ou encore la représentation parlementaire). Plus les valeurs de cet indice sont élevées, plus l'égalité entre les sexes est grande.

Le pourcentage de femmes parmi les diplômés en STIM données pour la période 2012-15) indique la proportion de femmes qui ont obtenu un diplôme universitaire en STIM parmi l'ensemble des diplômés de ces domaines.

La performance relative, ou point fort relatif, dans PISA 2015 mesure la performance d'un élève dans un domaine d'évaluation (mathématiques, sciences ou compréhension de l'écrit) par rapport à sa performance moyenne dans l'ensemble de ces trois principaux domaines d'évaluation. Les élèves forts en sciences auront une performance relative positive dans ce domaine d'évaluation (c'est-à-dire une performance en sciences supérieure à la performance moyenne dans les trois domaines d'évaluation). Tous les scores ont d'abord été standardisés au sein de chaque pays, de sorte qu'ils sont exprimés dans des unités correspondant à la variation globale (écart-type) observée dans chaque pays. 
Égalité et différences entre les sexes en termes de performance relative en sciences, et pourcentage de femmes parmi les diplômés en STIM

a)

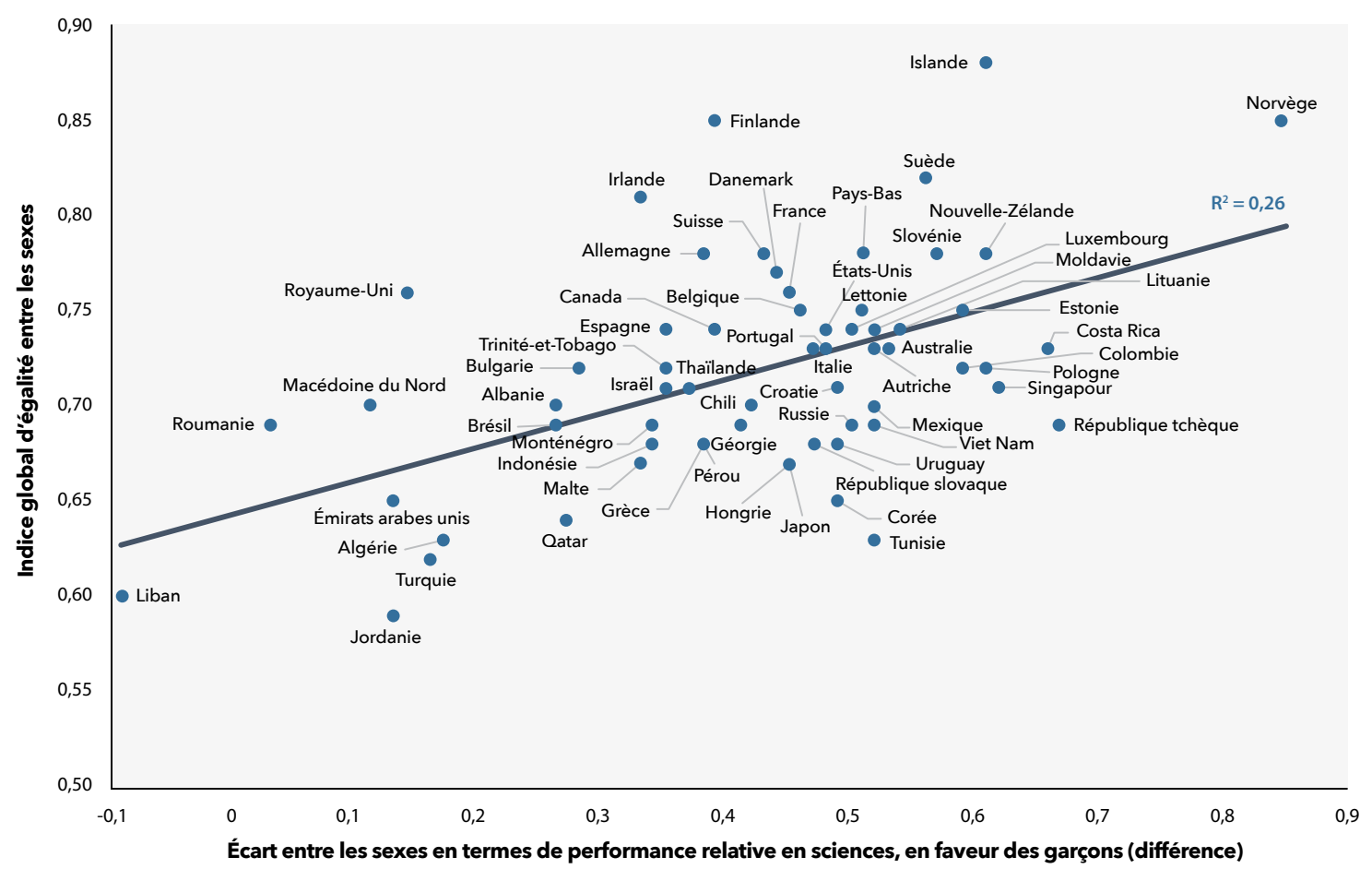

b)

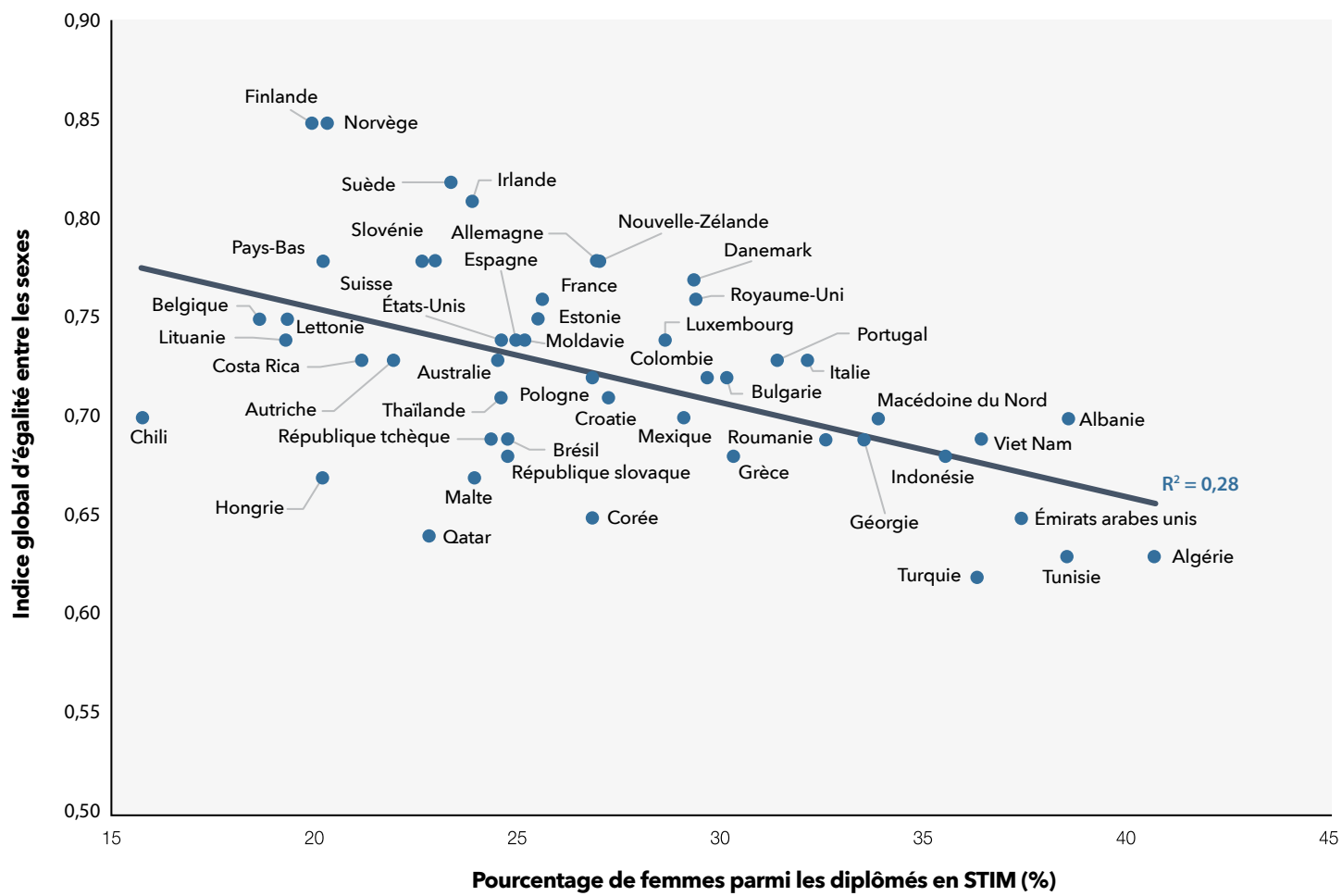

Source : G. et D.C. Geary (2018), "The Gender-Equality Paradox in Science, Technology, Engineering, and Mathematics Education ", Psychological Science, 29/4, pp. 581-593, https://doi.org/10.1177/0956797617741719. 
II est intéressant de noter que dans les pays où l'égalité hommes-femmes est plus grande, comme la Norvège et la Suède (soit ceux affichant des valeurs plus élevées sur l'indice global d'égalité entre les sexes), l'écart entre les sexes de performance relative en sciences, en faveur des garçons, est plus marqué, et les femmes sont moins nombreuses parmi les diplômés en STIM que dans les pays où le niveau d'égalité entre les sexes est plus faible.

\section{Les garçons affichent un niveau plus élevé de confiance en leurs capacités en sciences et d'intérêt pour les sujets scientifiques.}

L'enquête PISA montre aussi qu'en 2015, l'efficacité perçue en sciences était plus forte chez les garçons que chez les filles dans 39 des 67 pays et économies participants. De même, dans 51 pays/économies, les garçons faisaient part d'un plus grand intérêt que les filles pour les sujets scientifiques. Là encore, l'analyse montre que ces écarts entre filles et garçons d'efficacité perçue et d'intérêt sont particulièrement marqués dans les pays affichant une plus grande égalité entre les sexes.

Ces différences de performance relative, d'efficacité perçue, de plaisir et d'intérêt pour les sciences entre garçons et filles expliquent en grande partie l'infériorité des taux d'obtention d'un diplôme en STIM chez les femmes.

\section{Différence d'efficacité perçue en sciences entre les sexes}

et indice global d'égalité entre les sexes

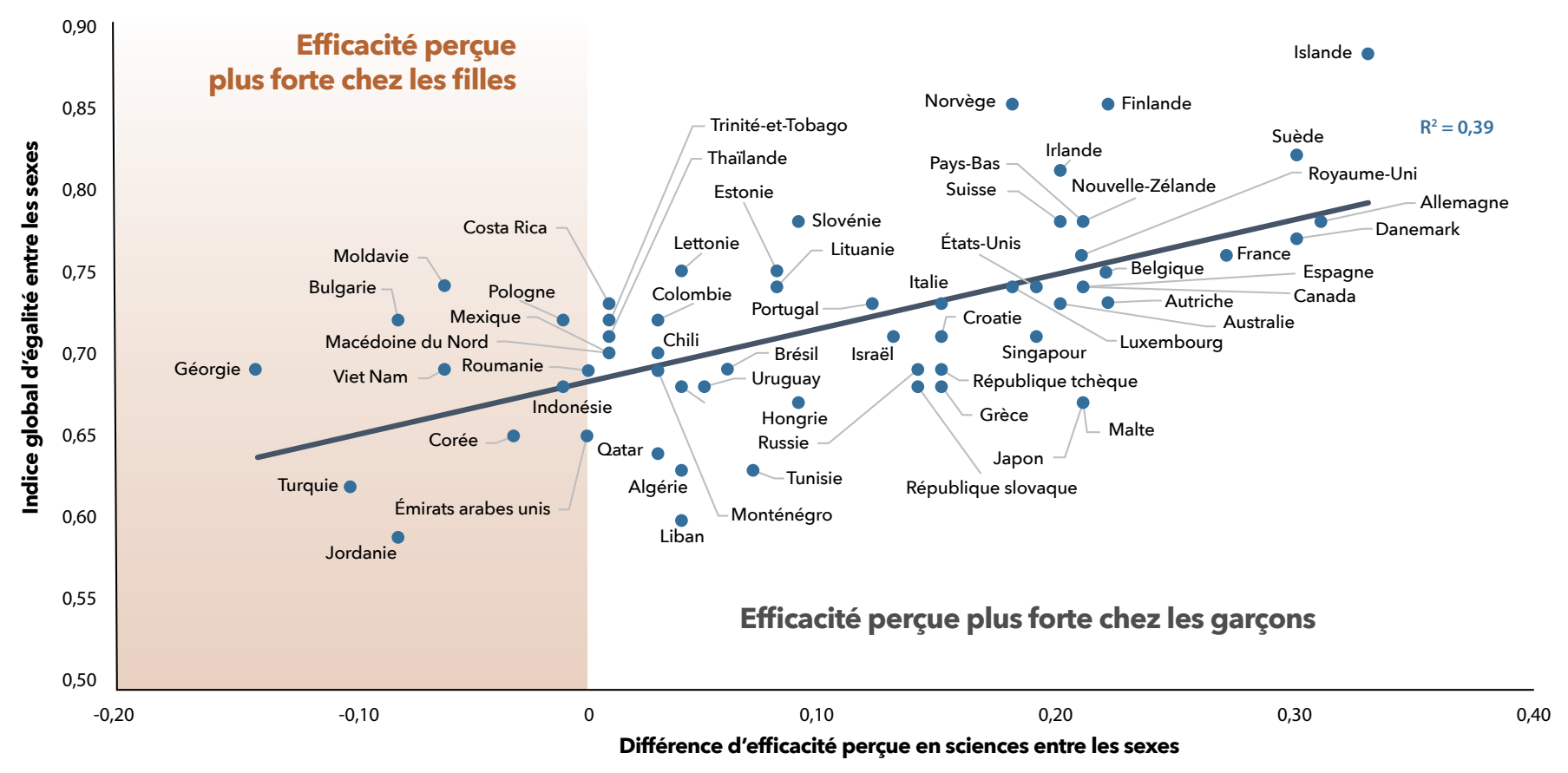

Source : G. et D.C. Geary (2018), «The Gender-Equality Paradox in Science, Technology, Engineering, and Mathematics Education », Psychological Science, 29/4, pp. 581-593, https://doi.org/10.1177/0956797617741719

\section{D’après les données PISA, un grand nombre de filles ont le profil pour embrasser une carrière dans les domaines des STIM mais peu d'entre elles le font dans les faits.}

Les auteurs utilisent différents critères de compétence et d'attitudes en sciences, d'après les données de l'enquête PISA 2015, pour calculer le pourcentage de filles ayant le profil pour obtenir un diplôme universitaire en STIM parmi l'effectif total d'élèves. Les résultats révèlent que les filles sont bien plus nombreuses parmi les élèves atteignant le niveau 4 sur les échelles PISA de compétence dans l'ensemble des trois principaux domaines d'évaluation (49 \%, en moyenne, dans les 67 pays et économies participants) que les femmes parmi les diplômés universitaires en STIM entre 2012 et 2015 (28 \%, en moyenne). En outre, lorsque les auteurs restreignent encore la catégorie d'élèves susceptibles d'obtenir un diplôme universitaire en STIM aux élèves très performants dont le plaisir, l'intérêt et l'efficacité perçue en sciences se situent dans la moitié supérieure de la distribution internationale de ces attitudes, les filles représentent alors $41 \%$ de cette catégorie. 
La différence entre les pourcentages escompté et effectif de femmes parmi les diplômés en STIM se réduit considérablement lorsque les auteurs restreignent encore leur définition des élèves ayant le profil pour réussir un cursus universitaire en STIM à ceux affichant un point fort relatif en sciences et en mathématiques, plutôt qu'en compréhension de l'écrit. Sur la base de cette définition, seule une fille sur trois (34 \%) a le profil pour obtenir un diplôme en STIM. Toutefois, dans la plupart des pays, le pourcentage de femmes diplômées en STIM reste inférieur à celui escompté.

\section{Pourcentage de filles de 15 ans ayant le profil pour obtenir un diplôme universitaire en STIM et de femmes y parvenant effectivement}

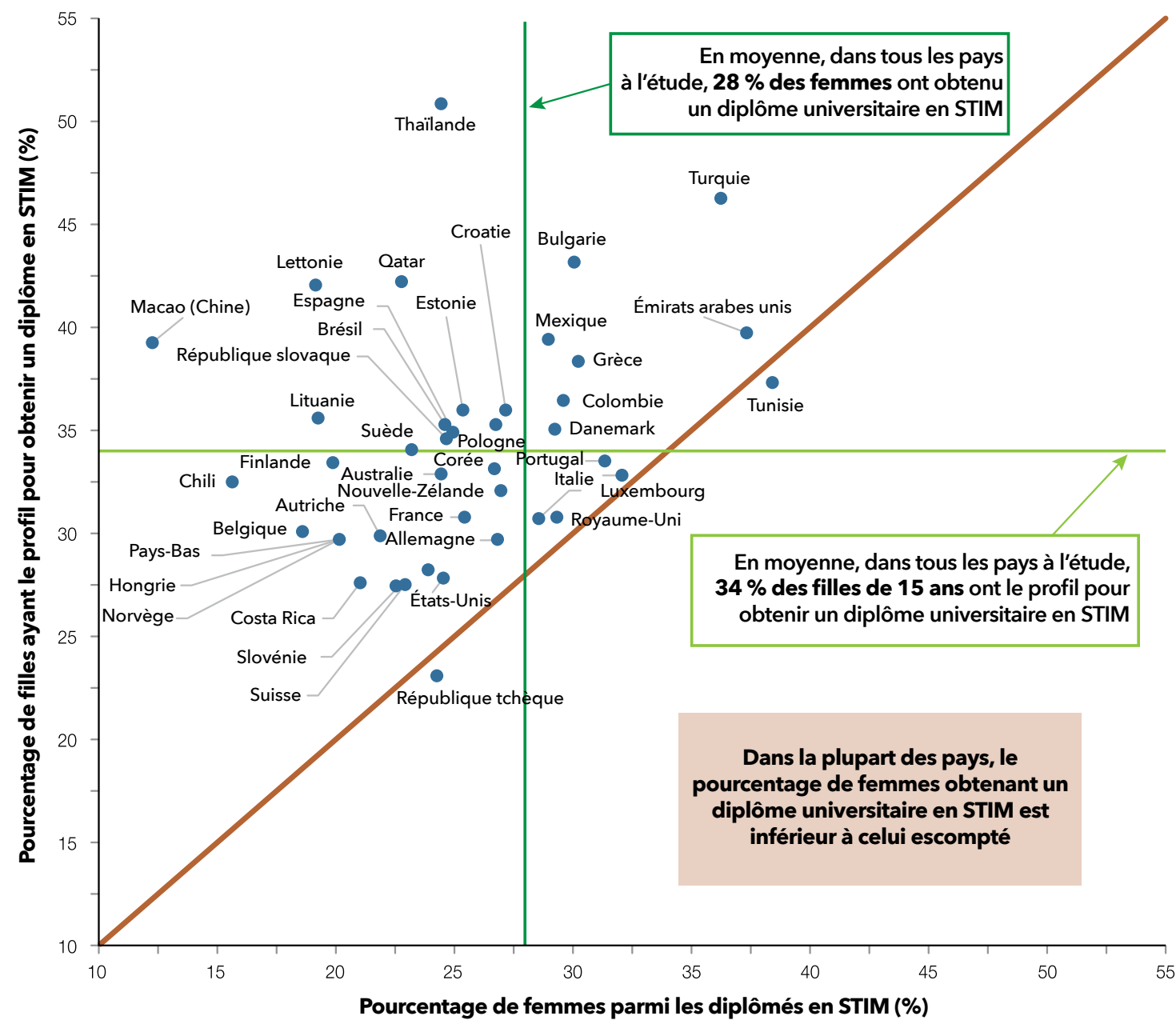

Remarque : Par filles ayant le profil pour obtenir un diplôme universitaire en STIM, on entend celles qui se situent au niveau 4 sur l'échelle PISA de compétence en sciences et dans la moitié supérieure de la distribution internationale du plaisir, de l'intérêt et de l'efficacité perçue en sciences.

Source : G. et D.C. Geary (2018), « The Gender-Equality Paradox in Science, Technology, Engineering, and Mathematics Education », Psychological Science, 29/4, pp. 581-593, https://doi.org/10.1177/0956797617741719

\section{Pour conclure}

Les travaux de Stoet et Geary (2018) semblent indiquer que les élèves pourraient être influencés dans leurs choix de carrière par leur perception de leur point fort scolaire relatif, ainsi que par leur confiance en leurs capacités en sciences et leur intérêt pour ce domaine. Contrairement à de nombreux garçons très performants, nombre de filles très performantes peuvent ne pas embrasser une carrière scientifique, même si elles ont toutes les capacités pour y parvenir, car elles sont également susceptibles de compter parmi les meilleurs de la classe dans des matières non scientifiques. Par conséquent, pour garantir une meilleure représentation des femmes dans les carrières scientifiques, il peut s'avérer tout aussi important de lutter contre la sous-performance des garçons en compréhension de l'écrit que de soutenir la performance des filles dans les matières en rapport avec les STIM et leurs attitudes à leur égard. 


\section{Pour tout complément d'information}

Contacter : Tarek Mostafa (tarek.mostafa@oecd.org)

Consulter : Stoet, G. and D.C. Geary (2018), "The Gender-Equality Paradox in Science, Technology, Engineering, and Mathematics Education", Psychological Science, 29/4, 581-593, https://doi.org/10.1177/0956797617741719

Le mois prochain : La scolarisation en zone rurale a-t-elle une incidence sur les contenus et les modalités d'apprentissage?

Ce document est publié sous la responsabilité du Secrétaire général de l'OCDE. Les opinions et les arguments exprimés ici ne reflètent pas nécessairement les vues officielles des pays membres de l'OCDE.

Ce document, ainsi que les données et cartes qu'il peut comprendre, sont sans préjudice du statut de tout territoire, de la souveraineté s'exerçant sur ce dernier, du tracé des frontières et limites internationales, et du nom de tout territoire, ville ou région.

Les données statistiques concernant Israël sont fournies par et sous la responsabilité des autorités israéliennes compétentes. L'utilisation de ces données par l'OCDE est sans préjudice du statut des hauteurs du Golan, de Jérusalem-Est et des colonies de peuplement israéliennes en Cisjordanie aux termes du droit international.

Ce texte est disponible sous licence Attribution - Pas d'Utilisation Commerciale - Partage dans les Mêmes Conditions 3.0 Organisations Internationales (CC BY-NCSA 3.0 IGO). Pour toute information spécifique quant à l'étendue et aux termes de la licence ainsi que d'une possible utilisation commerciale de ce texte ou pour tout usage de données PISA, prière de consulter les Conditions d'utilisation sur http://www.oecd.org/fr/conditionsdutilisation. 